\section{O PARADIGMA TECNOLÓGICO DA INTERNET DAS COISAS E SUA RELAÇÃO COM A CIÊNCIA DA INFORMAÇÃO}

\author{
Vitor Rozsa* \\ Moisés Lima Dutra* \\ Adilson Luiz Pinto \\ Enrique Muriel-Torrado
}

RESUMO Diante do ininterrupto avanço tecnológico dos últimos anos, o paradigma da Internet das Coisas (loT) progrediu e atualmente é capaz de propor soluções para desafios globais. O principal insumo utilizado pelas aplicações baseadas na loT é a informação, sendo gerada de forma constante e a partir de diferentes fontes, suscitando questões relacionadas a coleta, armazenamento, representação, recuperação e uso. Em vista destas questões, esta pesquisa busca identificar as possíveis relações existentes entre a Ciência da Informação $(\mathrm{Cl})$ e a loT, de forma a compreender como o campo da $\mathrm{Cl}$ pode contribuir para o desenvolvimento da loT. Esta pesquisa configura-se como exploratória e qualitativa. A relação entre $\mathrm{Cl}$ e loT é explorada por meio da análise do conceito de informação no contexto da loT; da identificação de especialidades da $\mathrm{Cl}$ que possam gerar soluções que contribuam para a evolução do paradigma da loT; e de como as tecnologias da loT podem ser utilizadas em vista de problemas informacionais. A partir disso, foi possível perceber a relevância da informação trabalhada na loT para a $\mathrm{Cl}$ e a para a sociedade, bem como as oportunidades para a contribuição das diferentes especialidades do campo da $\mathrm{Cl}$ no desenvolvimento do paradigma da loT.

Palavras-chave: Internet das Coisas. Ciência da Informação. Informação como coisa. Objetos inteligentes.
* Graduado em Sistemas de Telecomunicações pelo Instituto Federal de Santa Catarina, Brasil. Mestrando no Programa de Pós-Graduação em Ciência da Informação da Universidade Federal de Santa Catarina, Brasil.

E-mail: vitor.rozsa@hotmail.com.

** Doutor em Computação pela Universidade de Lyon I, França. Docente permanente do Programa de Pós-Graduação em Ciência da Informação da Universidade Federal de Santa Catarina, Brasil.

E-mail: moises.dutra@ufsc.br.

*** Doutor em Documentação pela Universidad Carlos III de Madrid, Espanha. Docente permanente do Programa de Pós-Graduação em Ciência da Informação da Universidade Federal de Santa Catarina, Brasil.

E-mail: adilson.pinto@ufsc.br.

**** Doutor em Documentação pela Universidad de Granada, Espanha. Docente permanente do Programa de Pós-Graduação em Ciência da Informação da Universidade Federal de Santa Catarina, Brasil.

E-mail: enrique.muriel@ufsc.br

\section{INTRODUÇÃO}

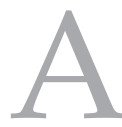
Internet das Coisas (IoT - do inglês Internet of Things) é um paradigma tecnológico que realiza a interconexão entre os mundos físico e digital, criando um "meio informacional" que aumenta a percepção das pessoas sobre o cenário ao seu redor. No contexto da IoT, pessoas e objetos inteligentes estão conectados na Internet em qualquer lugar e a todo momento, produzem e consomem informações e são capazes de comunicarem-se entre si. Este paradigma exige cada vez mais atenção, uma vez que está em constante expansão e baseia-se principalmente na produção e consumo de informações que devem ser operacionalizadas.

A IoT vem ganhando atenção e importância no mercado mundial ao longo da última década. É esperado que o mercado da IoT crescerá de US\$ 591,7 bilhões em 2014 para US\$ 1,3 trilhões até 2019 (VERIZON, 2016). Para 2025, a estimativa do potencial econômico da IoT varia entre US\$4 trilhões - no pior caso - e US\$ 11 trilhões - no melhor caso (MCKINSEY GLOBAL INSTITUTE, 2015). Do ponto de vista da sociedade como um todo, o benefício na adoção 
das tecnologias da IoT está no potencial existente para gerar soluções que abordem desafios de importância global (ITU; CISCO, 2016).

Podemos identificar a aplicação das tecnologias da IoT e a geração de soluções para desafios em diferentes contextos. A IoT é empregada em uma grande diversidade de cenários como, por exemplo, na agricultura (CAO-HOANG; DUY, 2017; QIN; LU; ZHU, 2015), logística (CHO; KIM, 2017; QU et al., 2016), indústria (KAHN et al., 2017; USMAN; ABBAS, 2014), transporte (PUIU et al., 2017; MAINETTI et al., 2015), construções (LI, 2017; RUSSELL; RUSSELL; GOUBRAN; KWAMENA, 2015), ambiente (ZAMBRANO et al., 2017; GUO et al., 2015; LIU et al., 2014) e cuidados médicos (YOO; CHUNG, 2017; MA et al., 2015). Destacamos que a viabilidade dos cenários da IoT depende da contínua integração/conexão de objetos do cotidiano na Internet (GUBBI et al., 2013) e, com isto, do aperfeiçoamento de nosso ambiente tornando-o mais capaz de lidar com situações habituais de forma autônoma e inteligente.

O paradigma da IoT está apto a causar um grande impacto em nossa sociedade, uma vez que os objetos inteligentes podem ser facilmente integrados nos serviços existentes (NAITO, 2017). Estes objetos são capazes de coletar grandes quantidades de dados sobre as pessoas e o ambiente e, assim, poderem responder de maneira autônoma a determinadas situações ou disponibilizar informações que aumentam a percepção das pessoas sobre o contexto ao seu redor. Diante disso, podemos deduzir que o insumo principal na IoT é a informação, que é gerada e consumida tanto pelas pessoas quanto pelos objetos "inteligentes".

Atualmente no mundo há uma crescente quantidade de dispositivos conectados que produzem e consomem informações. De acordo com dados da IDC (2015), em 2015 havia no mundo por volta de 13 bilhões de dispositivos conectados na Internet. Ainda segundo a mesma fonte, até 2020 projeta-se uma quantidade de 30 bilhões de dispositivos conectados. Somados, estes dispositivos produzem quantidades massivas de informações que podem ser exploradas para gerar valor para as pessoas e negócios. Porém, neste contexto surgem algumas questões que primeiramente precisam ser respondidas (HOSAIN, 2016): Como estes dados serão armazenados? Como serão analisados? Como serão transmitidos? Como serão buscados e recuperados?
Em virtude da grande quantidade de dados gerados na IoT, provenientes de diversas fontes e sobre diferentes eventos, surge a necessidade da gestão desses dados para que possam ser utilizados em benefício das pessoas. Para Santos e Vidotti (2009), um dos papéis da Ciência da Informação $(\mathrm{CI})$ é converter o grande volume de dados operacionais disponíveis diariamente em informações consistentes que permitam a tomada de decisões e agreguem valor às atividades diárias e negócios. Diante do meio informacional criado pela IoT, no qual potencializa-se o acesso à informação e seu consumo pelas pessoas de forma direta ou indireta ${ }^{1}$, a CI desempenha papel relevante ao investigar o tratamento dos dados e sua conversão em informações úteis às pessoas nos cenários da IoT.

$\mathrm{Na}$ IoT, a questão preponderante está relacionada à conversão de dados capturados em informação para gerar conhecimento e proporcionar ambientes mais convenientes para as pessoas (TSAI et al., 2014). Em pesquisa realizada por Whitmore, Agarwal e Xu (2015) identificou-se que os trabalhos sobre a IoT estão mais focados em questões tecnológicas. Uma grave consequência do foco exclusivo em tecnologias é o risco de excluir-se o fator humano do contexto em que estas tecnologias serão utilizadas (ITU; CISCO, 2016). Nesse sentido, o interesse da CI no estudo e compreensão de ferramentas e fatores - tanto humanos quanto tecnológicos - relacionados à origem, coleta, organização, armazenamento, recuperação, interpretação, transmissão, transformação e uso de informação justifica uma estreita relação com o paradigma tecnológico da IoT.

Diante da relevância social e do foco na informação manifestado pelo paradigma tecnológico da IoT, esta pesquisa busca identificar as possíveis relações entre a CI e a IoT. A principal questão a ser respondida é "Como o campo da CI pode contribuir para o desenvolvimento da IoT"? Para isto, os objetivos desta pesquisa são: explorar a relação entre CI e IoT por meio da análise do conceito de informação no contexto da IoT; identificar especialidades da CI que possam gerar soluções que ajudem a evoluir o paradigma da IoT; e identificar como as tecnologias da IoT podem ser utilizadas em vista de problemas

\footnotetext{
O consumo de informações de forma indireta se refere à tomada de decisões de forma automática por objetos inteligentes.
} 
informacionais. Assim, espera-se gerar subsídios para o desenvolvimento de pesquisas em CI no contexto da IoT.

Esta é uma pesquisa exploratória, pois tem em vista compreender como a CI e o paradigma tecnológico da IoT estão correlacionados. Também se configura como uma pesquisa qualitativa e de natureza básica. Utilizaram-se as bases de dados Scopus, IEEE Xplore e ACM na pesquisa bibliográfica.

O restante dessa pesquisa está estruturado da seguinte forma. Na seção 2, descrevemos em maiores detalhes questões referentes à IoT. Na seção 3, discutimos sobre o caráter especial da informação em cenários IoT, a partir do ponto de vista da CI. Na seção 4, descrevemos áreas de pesquisa em CI potencialmente beneficiárias da IoT. E, por fim, na seção 5 são apresentadas as considerações finais desta pesquisa e sugestões de possíveis rumos para investigações futuras.

\section{INTERNET DAS COISAS}

O paradigma tecnológico da IoT está se tornando cada vez mais maduro. Em 1999, Kevin Ashton, cofundador do Auto-ID Center do Massachusetts Institute of Technology (MIT), utilizou pela primeira vez o termo "Internet of Things", quando a visão da IoT ainda estava baseada fundamentalmente no uso da tecnologia de identificação por radiofrequência (do inglês, Radio-Frequency IDentification - RFID). Passadas então quase duas décadas, o conjunto de tecnologias abrangidas e de cenários de aplicação atendidos expandiu-se consideravelmente.

Atualmente, a IoT pode ser considerada a nova geração tecnológica que pode elevar nosso campo tecnológico a novos patamares (SRI; PRASAD; VIJAYALAKSHMI, 2016). Se considerarmos que o advento da Internet levou à interconexão em massa entre pessoas, a próxima revolução será a de objetos interconectados para criar ambientes inteligentes (GUBBI et al., 2013). As tecnologias empregadas na IoT para alcançar estas visões são diversas, tais como: sensores para deteç̧ão de eventos; protocolos de comunicação; organização, armazenamento, representação e recuperação de informações; fusão e processamento de dados; entre outras.

De modo a melhor compreender este paradigma tecnológico, e assim estarmos aptos a identificar pontos de interseção com a CI, realizamos aqui uma breve revisão sobre as principais características da IoT. Entre estas características estão a arquitetura e constituição da IoT e seus cenários de aplicação.

\section{I Arquitetura e constituição}

A arquitetura de uma aplicação na IoT define a forma como as tecnologias interagem para fornecer serviços ao usuário. As principais tecnologias que constituem estas aplicações estão relacionadas a identificação, sensoriamento, comunicação, computação, serviços e semântica (AL-FUQAHA et al., 2015). Além disso, o relacionamento entre as tecnologias pode nos fornecer pistas sobre como a informação é utilizada nos sistemas baseados na IoT.

Podemos visualizar a arquitetura da IoT como uma pilha de camadas na qual cada camada é construída sobre camada anterior. Atualmente, existem diferentes arquiteturas que podem ser utilizadas na construção de aplicações para a IoT (RAY, 2016), sendo a mais comum baseada em três camadas (YANG et al., 2011; TSAI et al., 2014; ABDMEZIEM; TANDJAOUI; ROMDHANI, 2015; AL-FUQAHA et al., 2015), que são: camada de percepção; camada de rede; e camada de aplicação. Esta arquitetura e sua composição está representada na Figura a seguir.

Figura 1: Arquitetura de uma aplicação IoT baseada em três camadas

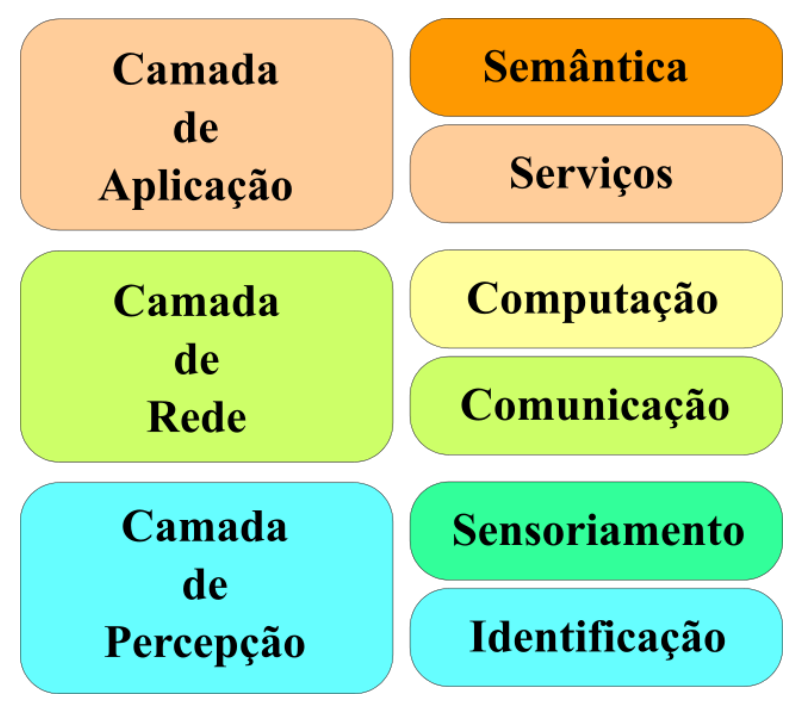

Fonte: Elaborado pelos autores 
A camada de percepção é responsável por transformar as informações no ambiente em sinais digitais para serem transmitidos na rede. Podemos entender que as tecnologias de identificação e sensoriamento pertencem a esta camada. A identificação se refere às tecnologias utilizadas para viabilizar o discernimento e comunicação entre os objetos inteligentes, enquanto o sensoriamento se refere tanto aos dispositivos utilizados na coleta de informações quanto aos dispositivos utilizados para interagir (i.e. atuadores) com o ambiente.

Rozsa et al. (2016) identificaram 5 principais conjuntos de sensores utilizados pelas aplicações na IoT, agrupados de acordo com seus tipos de informações coletadas: (a) movimento; (b) posição; (c) ambiente; (d) medida de massa, e; (e) biosensores. Os mesmos autores também destacam a relevância de tecnologias como o RFID e Global Positioning System (GPS) que, apesar de não se tratarem de sensores, são frequentemente utilizadas nas aplicações da IoT, principalmente para a identificação e localização de objetos e/ou pessoas.

A camada de rede realiza a interconexão entre as camadas de sensoriamento e aplicação e viabiliza a troca de informações entre os objetos inteligentes. Além disso, a camada de rede também é responsável pelo processamento e armazenamento das informações coletadas, para posterior uso pela camada de aplicação. A computação em nuvem é considerada como uma tecnologia elementar em vista da gestão de dados na IoT (ABDMEZIEM; TANDJAOUI; ROMDHANI, 2015) e para viabilizar aplicações futuras baseadas em Big Data e aprendizagem de máquina. Sendo assim, nesta camada estão inclusas as tecnologias de computação e comunicação. As tecnologias de computação se referem aos componentes de software que abstraem o hardware e fornecem o ambiente de execução das aplicações; e aos componentes de hardware, correspondentes às plataformas sobre as quais as aplicações de software executam. Já as tecnologias de comunicação são responsáveis pela disseminação de informações entre as diferentes camadas.

Por fim, a camada de aplicação se refere ao consumo da informação provida pela camada de rede. Nesta camada unem-se as informações obtidas nos cenários com o objetivo de disseminálas e prover serviços nos variados domínios de aplicação da IoT. Nesta camada estão inclusas as tecnologias de serviços e semântica. Os serviços se referem a diferentes tipos de sistemas que podem ser entendidos como elementos intermediários que abstraem as tecnologias de baixo nível - e possíveis heterogeneidades existentes - proporcionando transparência de acesso à estas tecnologias. A semântica está relacionada principalmente à capacidade de os objetos inteligentes "compreenderem" inequivocamente o contexto no qual estão inseridos, sendo as ontologias e os padrões da Web Semântica as principais ferramentas utilizadas para alcançar este propósito.

\subsection{Cenários de aplicação}

Devido ao caráter situacional da informação na IoT podemos imaginar diferentes aplicações para uma infinidade de contextos. Para isso, basta que sejam reconhecidos quais são os problemas que pretendemos resolver em determinado cenário e que sejam identificados quais tipos de informações são úteis e passíveis de coleta pelos sensores neste cenário. Dessa forma, perpetuam-se as aplicações da IoT em praticamente todas as áreas do cotidiano das pessoas, em empreendimentos e na sociedade como um todo (CHETAN; TEJASWINI, 2016).

Os cenários de aplicação na IoT podem ser categorizados dentro de domínios, sendo oportuno notar que existem diferentes categorizações geradas por diferentes autores. Por exemplo, Borgia (2014) fornece uma taxonomia de cenários de aplicação da IoT dividida em domínios, áreas de aplicação e cenários. Segundo a autora, os principais domínios da IoT são (i) indústria, (ii) cidades inteligentes e (iii) saúde e cuidados médicos, com cada domínio comportando uma grande diversidade de cenários de aplicação que podem ser beneficiados pelas tecnologias da IoT. A seguir, são descritos em maiores detalhes possíveis cenários da IoT para cada um destes domínios.

\subsection{Monitoramento de equipamentos pesados}

$\mathrm{Na}$ indústria, podemos encontrar, por exemplo, aplicações voltadas para o monitora- 
mento e controle de equipamentos pesados. Estes tipos de aplicações buscam manter um exame constante das condições de funcionamento dos equipamentos, visando identificar previamente possíveis problemas de funcionamento e necessidade de manutenção nestes equipamentos. Por exemplo, a ABB, fornecedora líder de equipamentos para mineração (ABB, 2017), oferece estes tipos de aplicações em seus produtos.

Em seu moinho movido por campo magnético, a $A B B$ permite a coleta de diferentes informações sobre sua operação para a predição de possíveis problemas. Por meio destas informações, é possível identificar com até oito dias de antecedência quando determinado moinho provavelmente irá falhar, o que implicaria na interrupção das operações de moagem (ABB, 2017). Estando ciente disso, os responsáveis podem decidir por realizar manutenções emergenciais de modo que o equipamento esteja apto a operar até a próxima manutenção programada.

\subsubsection{Sensoriamento urbano}

Em um esforço conjunto entre o laboratório de pesquisas Argonne e a Universidade de Chicago, desenvolveu-se o projeto de sensoriamento urbano denominado Matriz de Coisas (do inglês, Array of Things AoT). O AoT é um equipamento a ser instalado em diferentes localidades em uma cidade inteligente e que monitora diversos aspectos que impactam nas condições de vida das pessoas (ARRAY OF THINGS, 2016). Este equipamento é capaz de monitorar condições ambientais (ex.: temperatura, umidade, pressão e ruídos), a qualidade do ar e a presença de veículos e pessoas em determinado local. Estas informações são disponibilizadas gratuitamente na Web para serem utilizadas pelo público.

Estas informações sobre a cidade podem ser utilizadas em aplicações diversas. Entre as aplicações sugeridas pelo projeto estão (ARRAY OF THINGS, 2016): a) por meio dos monitores de condições ambientais, identificar os horários do dia mais saudáveis para a realização de caminhada e as rotas mais saudáveis; b) por meio de câmeras no AoT, identificar locais de alagamento para orientar os serviços da cidade e evitar danos à infraestruturas e a proliferação de doenças; e c) por meio de sensores de presença, reconhecer áreas densamente ocupadas por pedestres ou veículos e identificar rotas alternativas ou sincronizar semáforos, amenizando o trânsito e a poluição causada por congestionamentos.

\subsubsection{Preservação de Relíquias Culturais}

Ainda nas cidades inteligentes, podemos encontrar diferentes situações nas quais objetos históricos são armazenados em depósitos, exibidos ao público em museus ou são transportados para diferentes museus por diferentes razões (XIAO et al., 2016) como, por exemplo, empréstimos, permutas e doações (COSTA, 2006). Estes objetos possuem valor cultural alto, pois representam a herança histórica de determinada região ou país e precisam ser gerenciados corretamente para que sejam preservados de forma eficiente e efetiva. Nesse contexto, tecnologias como o RFID e sensores podem ser empregados para prover informações que auxiliem no monitoramento e controle destes objetos.

Em vista da gestão inteligente e eficiente assim como da preservação de relíquias culturais, os autores Xiao et al. (2016) propuseram um sistema inteligente de gestão de relíquias culturais baseado no uso de RFID e sensores. Por meio da tecnologia RFID, as relíquias são ser identificadas e localizadas automaticamente, o que reduz erros (humanos) quando centenas de objetos precisam ser monitorados; e auxilia em sua proteção, uma vez que é possível identificar quando estes objetos estão sendo movidos de local indevidamente. Por meio de sensores de temperatura e umidade, é possível monitorar e ajustas as condições ambientais nas quais os objetos se encontram, aumentando assim sua preservação. É interessante notar que, neste sistema, o consumo das informações de temperatura e umidade é realizada por pessoas, que devem interpretar os dados e ajustar manualmente as configurações ideais nos atuadores (e.x.: ar-condicionado e umidificador). Porém este tipo de ação é executado convenientemente de forma automática na maioria dos sistemas baseados na IoT.

\subsubsection{Sistema de pâncreas artificial}

Na área da saúde, as aplicações podem estar relacionadas tanto com a parte de gestão das instituições médicas quanto com a avaliação 
e o monitoramento das condições de saúde dos pacientes. Nas instituições, as aplicações IoT podem ser utilizadas, por exemplo, para identificar a localização dos funcionários e para rastrear e identificar os materiais e equipamentos dentro do hospital. Para avaliação e monitoramento de pacientes, as aplicações IoT são utilizadas, por exemplo, para o diagnóstico ou acompanhamento das condições de saúde dos pacientes, e até mesmo para aplicação automatizada de medicamentos, como o Sistema de Pâncreas Artificial Aberto (do inglês, Open Artificial Pancreas System - OpenAPS).

O OpenAPS é uma aplicação projetada para monitorar e manter estável o nível de glicose no sangue de uma pessoa com diabetes (LEWIZ, 2017). Esta aplicação é composta por medidor de glicose, bomba de insulina, dispositivo de controle, bateria e dispositivo de comunicação. A aplicação é executada no dispositivo de controle, que periodicamente consulta o medidor de glicose e a bomba de insulina por novas informações sobre as condições do paciente. A partir destas informações, a aplicação determina se alguma ação é necessária e, em caso positivo, envia um comando para a bomba fornecer a dosagem apropriada de insulina ao paciente. Nessa configuração, a bateria alimenta o dispositivo de controle e o dispositivo de comunicação é utilizado para a coleta de informações e execução de comandos na bomba de insulina.

\section{A INFORMAÇÃO NA INTERNET DAS COISAS}

Podemos perceber que o principal insumo nas aplicações IoT é a informação, seja na forma de medidas sobre o funcionamento de equipamentos pesados, na forma de informações sobre diferentes condições de uma cidade, ou na forma de informações sobre as condições de saúde de uma pessoa. Porém, esta informação - a informação na IoT - contrasta com a informação tradicionalmente trabalhada na $\mathrm{CI}$, pois possui caráter especial que a aproxima da definição de "informação como coisa" de Buckland (1991), exigindo assim a realização de algumas considerações sobre esta questão antes de continuarmos.

A informação da IoT provém do ambiente, dos objetos do cotidiano, de animais, pessoas e até mesmo de eventos. Estas informações são diferentes daquelas tradicionalmente trabalhas no campo da $\mathrm{CI}$, no sentido de que não são conhecimentos gerados pela mente humana, tampouco estão armazenadas em documentos no sentido usual (ex.: banco de dados, textos, vídeos, etc). Elas estão a nossa volta para serem percebidas e internalizadas pelas pessoas. Assim como o conhecimento humano registrado é relevante para o desenvolvimento de novas pesquisas e tecnologias, a informação obtida por meio da IoT é relevante para, além disso, o dia a dia das pessoas nos mais variados cenários.

Julgamos que uma das interpretações do conceito de informação em CI que está mais próximo da informação da IoT é a informação como coisa de Buckland (1991). Em seu trabalho "Information as Thing", Buckland identifica a partir da literatura os 3 principais usos da palavra "informação": "informação como conhecimento"; "informação como processo"; e informação como coisa. Enquanto informação como processo, a informação se refere ao processo de apreensão da informação na mente humana. A informação como conhecimento se refere à mudança causada na informação como processo, ou seja, o produto da apreensão da informação na mente humana, o conhecimento. Por fim, a informação como coisa remete à objetos ao qual o termo "informação" é associado. Neste caso, as "coisas" ao nosso redor como objetos, textos, documentos e dados em bases de dados são consideradas informativas.

Basicamente, qualquer "coisa", seja um objeto, animal, pessoa ou evento, pode ser considerado informação como coisa (ou informativo), basta que alguém identifique um propósito para tal "coisa". Um exemplo prático dessa questão é o uso de "testemunhos de gelo" para estudar as condições climáticas em diferentes épocas. Um testemunho de gelo é uma amostra de gelo retirada da neve em diferentes profundidades. Estas amostras podem possuir bolhas de ar presas que fornecem pistas relevantes aos cientistas para o estudo sobre as condições atmosféricas de épocas passadas. Nesse contexto, é possível chegarmos à mesma conclusão de Buckland (1991), de que podemos entender qualquer coisa como informativa, bastando que alguém identifique alguma situação em que aquela "coisa" possui potencial informativo. Assim sendo, também podemos atribuir um caráter social à informação da IoT, no 
sentido de que depende tanto das experiências pessoais quanto do contexto social - do consenso - das pessoas para considerar algo como informativo.

\section{A RELAÇÃO ENTRE CIÊNCIA DA INFORMAÇÃO E INTERNET DAS COISAS}

De forma mais ampla e à parte de seu objeto, podemos explorar a relação entre CI e IoT por meio de duas perspectivas: (i) uma voltada para a IoT como ferramenta a ser empregada na melhoria ou solução de problemas informacionais; e (ii) outra voltada para a atuação do cientista da informação em vista de questões pertinentes à operacionalização da informação na IoT. A primeira perspectiva consiste na aplicação do paradigma da IoT de modo a melhorar processos de geração, acesso, coleta, disseminação e uso da informação em diferentes cenários. A segunda perspectiva envolve estudos mais aprofundados uma vez que se refere à proposição, por parte da CI, de soluções e formas de abordar os processos de informação no contexto da IoT.

O emprego das tecnologias da IoT em vista de problemas informacionais já ocorre independentemente da CI, mas também em cenários tradicionalmente relacionados a área. Exemplos podem ser observados em bibliotecas, museus e arquivos nos quais o uso da IoT é uma forma de facilitar o acesso às informações fornecidas pelas instituições; a interação dos usuários com o acervo; e a gestão e monitoramento das condições físicas dos acervos (XIAO et al., 2016; LI et al., 2016; CHENG et al., 2016; KORZUN et al., 2016; YAO et al., 2016; WANG; HU; CAI, 2011). Além disso, as tecnologias da IoT podem auxiliar no desenvolvimento de pesquisas científicas, por meio de sensores conectados para o monitoramento e coleta automatizada de dados. Este benefício é ressaltado por Brookes (1979, p.14 apud BUCKLAND 1991) quando menciona que a fonte primária de dados na ciência é a "observação de fenômenos naturais relevantes". As informações obtidas por meio da IoT também podem ser empregadas em benefício de sistemas de RI, de modo que as ferramentas de busca estejam melhor preparadas em relação às necessidades de informação do usuário, por meio da coleta de informações contextuais sobre o mesmo.

Por outro lado, a IoT configura-se como um paradigma tecnológico com amplas oportunidades para contribuição por parte da CI. A CI está habilitada a atuar em diversas etapas do ciclo de vida da informação na IoT como, por exemplo, na representação (ontologias, taxonomias, tesauros), armazenamento (ontologias e datasets), recuperação (ferramentas de busca e recuperação da informação), processamento (ontologias e Big Data) e uso (suporte à decisão e tomada de decisão) da informação.

A representação do conhecimento é uma questão primária na arquitetura da IoT. A organização, processamento e recuperação da informação de modo a gerar valor para o usuário são desafios na IoT (SRI; PRASAD; VIJAYALAKSHMI, 2016). A representação do conhecimento está diretamente ligada a estes aspectos, sendo as ontologias uma das principais ferramentas para esta finalidade. $\mathrm{O}$ cientista da informação pode contribuir com este quesito, por exemplo, por meio da elaboração de ontologias de domínio, tendo em vista os contextos (cenários) específicos das aplicações e seus propósitos. Convém destacar que a representação do conhecimento no cenário da IoT possui maior foco no processamento das informações de forma automatizada. Sendo assim, a ontologia deve ser modelada de forma que viabilize o processo de inferência para gerar novas informações úteis para serem consumidas pelas aplicações ou pessoas.

Outra aplicação da ontologia está relacionada à recuperação da informação. As informações a serem recuperadas podem referirse à arquitetura do sistema (ex.: informações sobre objetos inteligentes ou sensores), ao cenário (ex.: informações geradas pelos sensores ou sobre a operação das aplicações) e à aplicação (ex.: informações processadas pelas aplicações). Um grande obstáculo para a recuperação da informação no contexto da IoT é a quantidade massiva de informações geradas pelos diversos sensores e pela diversidade no formato dessas informações.

$\mathrm{Na}$ IoT encontramos cenários nos quais há um grande volume de dados sendo gerados a todo momento e que podem ser combinados com 
informações provenientes de diferentes fontes. Nestes cenários, o emprego de ferramentas do Big Data pode ser útil para a fusão das informações geradas pelos sensores como para análise descritiva, prescritiva e preditiva dessas informações (VERIZON, 2016). O próximo passo na evolução da IoT - que já está em andamento - é a Cognitive IoT. Esta evolução consiste em tornar as aplicações capazes de aprender a partir de suas experiências no ambiente, sendo o Big Data e a Aprendizagem de Máquina algumas das principais tecnologias para alcançar este objetivo.

A visualização da informação na IoT é igualmente uma questão de grande interesse, uma vez que podem ser utilizados meios diferentes dos tradicionais (ex.: computador pessoal ou dispositivo móvel) para este fim. Como interfaces de interação com o usuário na IoT podemos mencionar as superfícies inteligentes, em que praticamente qualquer superfície em uma construção inteligente pode tornar-se uma interface (LEE, 2016); interfaces para controle por voz (HAN et al., 2016; HUANG; LIU; ZHOU, 2015); e interfaces capazes de obter feedback visual do usuário, como os smart mirrors (MALLIK et al., 2015). Esta característica da IoT de suportar diferentes formas de interação com o usuário pode ser explorada pelo estudo da Arquitetura da Informação. O trabalho de Lacerda e Lima-Marques (2015) é pioneiro neste aspecto do ponto de vista da CI, sendo conveniente mencionar o destaque por parte dos autores sobre a relevância de considerar-se o aspecto humano na construção das aplicações da IoT, de modo que estas sejam adaptadas às pessoas ao invés das pessoas adaptarem-se às aplicações que lhes servem.

O impacto da informação da IoT no ambiente em que é coletada e utilizada também é um é aspecto que deve ser considerado. O meio informacional criado pela IoT pode ser visto como uma ecologia informacional. Está ecologia estaria constituída, por exemplo, pelas fontes de informação, produtores (objetos inteligentes) e consumidores (pessoas e objetos inteligentes) das informações. O consumo destas informações pode implicar em ações que impactem no ambiente gerando assim situações das quais novas informações serão coletadas.

Outra questão refere-se ao ciclo de vida da informação na IoT. A informação coletada na IoT é muitas vezes útil apenas no cenário no qual foi coletado e aplicável naquele cenário apenas dentro de uma determinada janela de tempo. Por exemplo, uma aplicação que utiliza o nível de incidência solar durante o dia para acionar o sistema de irrigação em um jardim não terá, a princípio, utilidade para informações sobre a incidência solar em dias anteriores. Desta maneira, podemos atribuir uma característica de efemeridade à informação da IoT. Por outro lado, ao invés do descarte das informações que "perderam sua validade", poderia pensar-se na aplicação de técnicas de Big Data tendo em vista prover uma funcionalidade de aprendizagem aos objetos inteligentes.

Por fim, facilitar o acesso a informações úteis nos ambientes em que as pessoas vivem é um dos propósitos da IoT. Espera-se que, por meio da interconexão de diferentes aplicações e tecnologias, sejam viabilizadas diferentes aplicações para o suporte à decisão (SHAH; YAQOOB, 2016), expectativa que na verdade atualmente atende-se até certo ponto. Por meio de uma infinidade de sensores que coletam informações sobre os mais variados aspectos da vida humana, pode-se auxiliar as pessoas a tomar decisões com maior precisão. Além disso, aplicações também podem executar ações automatizadas baseadas nestas informações. Entender de que forma o suporte à decisão pode ser melhorado e como a tomada de decisão automatizada deve ser realizada na IoT representam uma ampla área de estudo para a CI.

\section{CONSIDERAÇÕES FINAIS}

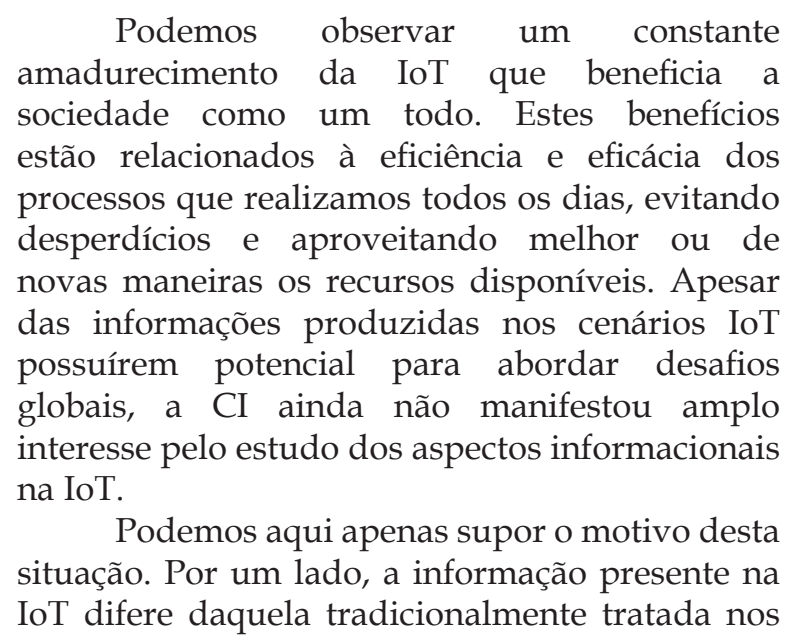


estudos de informação na CI. Por outro lado, o fenômeno da IoT tomou maior impulso apenas nos últimos anos, havendo somente recentemente apresentado oportunidades mais claras para uma intervenção construtiva por parte da CI. Não obstante, é evidente a contribuição do desenvolvimento deste paradigma tecnológico para a sociedade.

A CI pode contribuir no aprimoramento da IoT mas também pode-se empregar soluções da IoT em vista dos problemas informacionais que a CI busca resolver. Nesse sentido, o aprimoramento da IoT é duplamente benéfico, considerando que a CI está auxiliando no processo de desenvolvimento de uma ferramenta que ela mesma adota. A questão que deixamos aqui não é se os pesquisadores em CI devem se empenhar em desenvolver atividades relacionadas à IoT, mas sim, quais serão estas atividades.

Com esta pesquisa buscamos contribuir com os primeiros passos em direção ao estreitamento das relações entre CI e IoT. Explorou-se o conceito de informação na IoT do ponto de vista da CI utilizando-se a perspectiva de informação como coisa, de modo que podemos atribuir um caráter social à informação da IoT. Identificou-se soluções baseadas na IoT para problemas informacionais em cenários tradicionalmente trabalhados na $\mathrm{CI}$, como as bibliotecas, museus e arquivos. Por fim, explorou-se como a CI, por meio de suas especialidades, pode contribuir para a representação, armazenamento, recuperação, processamento e uso da informação no contexto da IoT.

O caminho para o estreitamento entre a CI e o paradigma tecnológico da IoT ainda é longo, porém, como visto, apresenta diversas perspectivas positivas. Como futuras pesquisas, sugere-se identificar de forma mais específica os possíveis temas nos quais a CI está apta a contribuir na IoT do ponto de vista de cada uma de suas especialidades.

Artigo recebido em 31/10/2017 e aceito para publicação em 30/I I/2017

\section{INTERNET OF THINGS FROM INFORMATION SCIENCE VIEWPOINT}

ABSTRACT As the Internet of Things (loT) paradigm keeps progressing, boosted by the continuous technological advance in recent years, it is now able to provide solutions to global challenges. The main input into loT-based applications is information. loT information are steadily generated from many sources and raise issues related to collecting, storing, representing, retrieving, and using. In the face of these issues, this work's main objective is to identify the possible relationship between Information Science (IS) and the loT, in order to understand how the IS field can contribute to the loT development. This is an exploratory and qualitative research. The relationship between IS and loT is researched by the analysis of the concept of information in IoT contexts; by the identification of IS specialties able to provide solutions that contribute to the evolution of the loT paradigm; and by the identification of how loT technologies can be used to tackle informational problems. In the end, it was possible for us to perceive the relevance of the loT-created information for IS and the society, as well as the existing opportunities for the application of different IS specialties in the development of the loT paradigm.

Keywords: Internet of Things. Information Science. Information-as-thing. Smart objects.

\section{REFERÊNCIAS}

ABB. Industrial IoT applications: Connected Mines. 2017. Disponível em: <http://new.abb. com/control-systems/features/industrial-IoTservices-people-use-cases $\geq$. Acesso em: 04 out. 2017.
ABDMEZIEM, M. R.; TANDJAOUI, D.; ROMDHANI, I. Architecting the Internet of Things: State of the Art. Robots And Sensor Clouds, [s.1.], p.55-75, 2015.

AL-FUQAHA, A. et al. Internet of Things: A Survey on Enabling Technologies, Protocols, and 
Applications. IEEE Communications Surveys \& Tutorials, [s.1.], v. 17, n. 4, p.2347-2376, 2015.

ARRAY OF THINGS. Home Page. 2016. Disponível em:< https://arrayofthings.github. io>. Acesso em: 04 out. 2017.

BORGIA, E. The Internet of Things vision: Key features, applications and open issues. Computer Communications, [s.1.], v. 54, p.1-31, 2014.

BROOKES, B. C. Theoretical informatics in stage II mechanization of IR processes. In International Federation for Documentation, Theoretical problems of informatics: New trends in informatics and its terminology. FID 568. Moscow: VINITI. p.10-18. 1979.

BUCKLAND, M. K. Information as thing. Journal of the American Society For Information Science, [s.1.], v. 42, n. 5, p.351-360, 1991.

CAO-HOANG, T.; DUY, C. N. Environment monitoring system for agricultural application based on wireless sensor network. Seventh International Conference on Information Science and Technology (ICIST), [s.1.], p.99-102, 2017.

CHENG, H. et al. Design and Implementation of Library Books Search and Management System Using RFID Technology. International Conference on Intelligent Networking and Collaborative Systems, [s.1.], p.392-397, 2016.

CHETAN, C.; TEJASWINI, N. P. Applications and Challenges of Internet-of-Things- A Survey. International Journal for Scientific Research \& Development, [s.1], v. 3, n. 11, p.521-524, 2016.

CHO, S.; KIM, J. Smart Logistics Model on Internet of Things Environment. Advanced Science Letters, [s.1.], v. 23, n. 3, p.1599-1602, 2017.

COSTA, E. P. Princípios Básicos da Museologia. Curitiba: Coordenação do Sistema Estadual de Museu. Secretaria de Estado da Cultura, 2006, 100p.

GUBBI, J. et al. Internet of Things (IoT): A vision, architectural elements, and future directions. Future Generation Computer Systems, [s.1.], v. 29, n. 7, p.1645-1660, 2013.
GUO, S. et al. The application of the Internet of Things to animal ecology. Integrative Zoology, [s.1.], v. 10, n. 6, p.572-578, 2015.

HAN, Y. et al. A smart home control system based on context and human speech. 18th International Conference On Advanced Communication Technology, [s.1.], p.165-169, 2016.

HOSAIN, S. Z. Reality Check: 50B IoT devices connected by 2020 - beyond the hype and into reality. 28 jun. 2016. Disponível em: <http:// www.rcrwireless.com/20160628/opinion/ reality-check-50b-iot-devices-connected-2020beyond-hype-reality-tag10>. Acesso em: 04 out. 2017.

HUANG, C.; LIU, A.; ZHOU, P. Using Ontology Reasoning in Building a Simple and Effective Dialog System for a Smart Home System. IEEE International Conference on Systems, Man, and Cybernetics, [s.1.], p.1508-1513, 2015.

IDC. Connecting the IoT: The Road to Success. Massachusetts. 08 jun. 2015. Disponível em:< http://www.idc.com/infographics/IoT>. Acesso em: 04 out. 2017

ITU; CISCO. Harnessing the Internet of Things for Global Development. Genebra, p.61, 2016.

KHAN, W. Z. et al. A reliable Internet of Things based architecture for oil and gas industry. 19th International Conference on Advanced Communication Technology (ICACT), [s.1.], p.705-710, 2017.

KORZUN, D. et al. Semantic infrastructure of a smart museum: toward making cultural heritage knowledge usable and creatable by visitors and professionals. Personal and Ubiquitous Computing, [s.1.], v. 21, n. 2, p.345-354, 2016.

LACERDA, F.; LIMA-MARQUES, M. Da Necessidade de Princípios de Arquitetura da Informação para a Internet das Coisas. Perspectivas em Ciência da Informação, Belo Horizonte, v. 20, n. 2, p.158-171, 2015.

LEE, T. This Device Will Turn Any Surface Into A Smart Surface. 18 maio 2016. Disponível em: 
http://www.ubergizmo.com/2016/05/knockiturns-surfaces-smart. Acesso em: 04 out. 2017.

LEWIZ, D. What is \#OpenAPS? - OpenAPS.org. 2017. Disponível em: <https://openaps.org/ what-is-openaps $>$. Acesso em: 04 out. 2017.

LI, D. et al. Design of Internet of Things System for Library Materials Management using UHF RFID. IEEE International Conference on Rfid Technology and Applications (rfid-ta), [s.l.], p.44-48, 2016.

LI, H. Design and implementation of smart home system under the framework of android and ZigBee technology. Agro Food Industry Hi-Tech. [s.1.], v. 28, n. 3, p.244-248, 2017.

LIU, J. et al. Software-defined internet of things for smart urban sensing. IEEE Communications Magazine, [s.1.], v. 53, n. 9, p.55-63, 2015.

MA, Y. J. et al. Health Internet of Things - Recent Applications and Outlook, Journal of. Internet Technology, [s.1] v. 16, n. 2, p.351-362, 2015.

MAINETTI, L. et al. A Smart Parking System based on IoT protocols and emerging enabling technologies. IEEE 2nd World Forum on Internet Of Things (wf-iot), [s.l.], 2015.

MALLIK, A. et al. Ontology based context aware situation tracking. IEEE 2nd World Forum on Internet of Things (wf-iot), [s.1.], 2015.

MCKINSEY GLOBAL INSTITUTE. The Internet of things: Mapping the value beyond the hype. p.144, 2015.

NAITO, K. A Survey on the Internet-of-Things: Standards, Challenges and Future Prospects. Journal of Information Processing, [s.1.], v. 25, p.23-31, 2017.

PUIU, D. et al. A public transportation journey planner enabled by IoT data analytics. 20th Conference on Innovations in Clouds, Internet and Networks (ICIN), [s.1.], p.355-359, 2017.

QIN, P.; LU, Z.; ZHU, T. Application Research on Agricultural Production Throughout the Internet of Things. Proceedings of the 2015 International
Conference on Management, Education, Information and Control, [s.l.], p.1219-1224, 2015.

QU, T. et al. System dynamics analysis for an Internet-of-Things-enabled production logistics system. International Journal of Production Research, [s.1.], v. 55, n. 9, p.2622-2649, 25 abr. 2016.

RAY, P. P. A survey on Internet of Things architectures. Journal of King Saud University - Computer and Information Sciences, [s.1.], p.129, 2016.

ROZSA, V. et al. An Application DomainBased Taxonomy for IoT Sensors. Advances in Transdisciplinary Engineering, Curitiba, v. 4, p.249-258, 2016.

RUSSELL, L.; GOUBRAN, R.; KWAMENA, F. Personalization Using Sensors for Preliminary Human Detection in an IoT Environment. International Conference on Distributed Computing in Sensor Systems, [s.1.], p.236-241, 2015.

SANTOS, P. L. V. A. C. S.; VIDOTTI, S. A. B. G. Perspectivismo e tecnologias de informação e comunicação: acréscimos à Ciência da Informação? DataGramaZero: Revista de Ciência da Informação, v.10, n.3, 2009.

SHAH, S. H.; YAQOOB, I. A survey: Internet of Things (IOT) technologies, applications and challenges. IEEE Smart Energy Grid Engineering, [s.1.], p.381-385, 2016.

SRI, S. T.; PRASAD, J. R.; VIJAYALAKSHMI, Y. A review on the state of art of Internet of Things. International Journal of Advanced Research in Computer and Communication Engineering, [s.1], v. 5, n. 7, p.189-193, 2016.

TSAI, C. et al. Data Mining for Internet of Things: A Survey. IEEE Communications Surveys \& Tutorials, [s.1.], v. 16, n. 1, p.77-97, 2014.

USMAN, M.; ABBAS, N. On the Application of IOT (Internet of Things) for Securing Industrial Threats. 12th International Conference on 
Frontiers of Information Technology, [s.1.], p.3740, 2014.

VERIZON. State of the Market: Internet of Things. New Jersey. p.24, 2016.

WANG, H.; HU, Q.; CAI, Y. Research on Network Platform of Ubiquitous Service in Ubiquitous Society: A Case Study on Archive. International Conference on Intelligence Science and Information Engineering, [s.l.], p.167-170, 2011.

WHITMORE, A.; AGARWAL, A.; XU, L. The Internet of Things - A survey of topics and trends. Information Systems Frontiers, [s.1.], v. 17, n. 2, p.261-274, 2015.

XIAO, C. et al. SCRMS: An RFID and Sensor Web-Enabled Smart Cultural Relics Management System. Sensors, [s.1.], v. 17, n. 1, p.60-81, 2016.
YANG, Z. et al. Study and application on the architecture and key technologies for IOT. International Conference on Multimedia Technology, [s.1.], p.747-751, 2011.

YAO, C. K. et al. IoT Application on Remote Monitoring and Control of Museum Display Cabinet. ICIC Express Letters Office. [s.l]. v. 10, n. 9, p.2249-2249, 2016.

YOO, H.; CHUNG, K. Heart rate variability based stress index service model using biosensor. Cluster Computing, [s.1.], p.1-11, 2017.

ZAMBRANO, A. M. et al. Technologies of Internet of Things applied to an Earthquake Early Warning System. Future Generation Computer Systems, [s.1.], v. 75, p.206-215, 2017. 